\title{
On a problem of infinite divisibility
}

\author{
Lev B. Klebanov ${ }^{a}$
}

\begin{abstract}
Let $f(t)$ be a characteristic function. The question on infinite divisibility of $g_{2 k}(t)=f^{(2 k)}(t) / f^{(2 k)}(0)$ is considered. There are given the condition for that function not to be infinite divisible. Some examples of infinite divisibility of $g_{2 k}(t)$ are given.
\end{abstract}

Key words: characteristic function; infinite divisibility; singularities

\section{Introduction}

Suppose that $X$ is a random variable possessing finite moment of even order $2 k$ and $f(t)$ is its characteristic function. Then $g_{2 k}(t)=f^{(2 k)}(t) / f^{(2 k)}(0)$ is a characteristic function again. In the case when $X$ is positive (or negative) random variable it is possible consider $g_{k}(t)=f^{(k)}(t) / f^{(k)}(0)$ when the $k^{t h}$ moment exists. An easy question arises. Under what conditions $g_{2 k}(t)$ is infinite divisible characteristic function? Clearly, it is possible to consider similar question not for a fixed $k$ only but for a finite or infinite set of such numbers. The aim of this publication is to consider these questions in some details and propose hypothetical answers.

\section{Negative results}

Let us start with a negative result.

${ }^{a}$ Department of Probability and Mathematical Statistics, Charles University, Prague, Czech Republic. e-mail: levbkl@gmail.com 
Theorem 2.1. Let $f(t)$ be a characteristic function of a random variable $X$ having a symmetric (around origin) distribution. Suppose that $\mathbb{E} X^{2 m}<\infty$ for an integer $m \geq 1$. Then the characteristic functions $g_{2 k}(t)=f^{(2 k)}(t) / f^{(2 k)}(0)$ $(k=1, \ldots, m)$ are not infinite divisible.

Proof. Consider the case $m=1$. Suppose the opposite. If $g_{2}(t)$ is infinite divisible it must be positive over the whole real line. Therefore, $f(t)$ must be concave on the whole real line. In this situation its graph should be under a tangent drawn to it at an arbitrary point $\left(t_{o}, f\left(t_{0}\right)\right)$. However, it contradicts to the boundness of $f(t)$. Therefore, $g_{2}$ has zeros and cannot be infinite divisible.

The similar arguments are applicable to each pair $g_{2 k-2}(t), g_{2 k}(t)$ for $k=1, \ldots, m$.

Obviously, the proof essentially uses the reality of symmetric characteristic function on the real line. However, the fact remains true for entire characteristic functions ${ }^{1}$. More precisely, the following result holds.

Theorem 2.2. Let $f(t)$ be entire characteristic function of non-degenerate distribution. No pair of the functions $g_{2 k}(t), g_{2 s}(t)(0 \leq k<s \leq m)$ may consists of infinitely divisible characteristic functions.

Proof. The statement is a simple consequence of the result by Tumura and Clunie (see [2]). Really, if $f(t)$ is entire characteristic function then all $g_{2 k}$ are entire functions. It is known (see, for example, [1]) the entire infinite divisible characteristic function has no zeros on the whole complex plain. From Theorem 3.8 [2] it follows that $f(t)=\exp (A t+B)$ in contradiction with non-degenerate character of the corresponding distribution.

From Theorem 2.2 it follows that characteristic functions of the normal and Poisson distributions do not possess the property of infinite divisibility of $g_{2 k}(t)$.

\section{Positive examples}

Here we give some examples of infinite divisible characteristic functions $f(t)$ for which one or more functions $g_{2 k}(t)(k>0)$ are infinite divisible too.

\footnotetext{
${ }^{1}$ For the definitions and properties see [1]
} 
Example 3.1. The main positive example is characteristic function of Gammadistribution

$$
f(t)=\frac{1}{(1-i a t)^{\gamma}}, \quad a>0, \quad \gamma>0,
$$

which is infinite divisible for all values of the parameters. It is clear that $g_{k}(t)$ is also characteristic function of the Gamma-distribution with different parameter $\gamma$ and therefore is infinite divisible. Note that $k$ is not supposed to be even number.

It is obvious that $f(t)$ is an analytic function in the strip $|\operatorname{Im} t|<1 / a$ and can be analytically prolonged outside of it, but is not a meromorphic for non-integer $\gamma$. The function $f(-t)$ possesses the same property as well.

The adding of a location parameter into the distribution with characteristic function $f(t)$ has no impact on its infinite divisibility, but it will change the form of $g_{2 k}(t)$.

Example 3.2. Suppose now that

$$
f(t)=\frac{\exp \{i b t\}}{1-i t}, \quad(b>0)
$$

is a characteristics of shifted exponential distribution (a particular case of shifted gamma-distribution). Then both $f(t)$ and $g_{2}(t)$ are infinite divisible.

Proof. To (3.2) corresponds characteristic function

$$
g_{2}(t)=\frac{e^{i b t}\left(2-2 i b(i+t)-b^{2}(i+t)^{2}\right)}{\left(2+2 b+b^{2}\right)(1-i t)^{3}} .
$$

We need to show (3.3) represent infinite divisible characteristic function. For the first glance it is not so because $g_{2}(t)$ has complex zeros. However, these zeros are outside of the strip of analyticity of $g_{2}(t)$, i.e. outside of the strip $|\operatorname{Im}(t)|<1$. The distributions with characteristic function $g_{2}$ is concentrated on semi-axis $x>b$. To verify infinite divisibility of $g_{2}$ let us use A.N. Kolmogorov representation of logarithm of the corresponding characteristic function:

$$
\log g_{2}(t)=i c t+\int_{-\infty}^{\infty}\left(e^{i t u}-1-i t u\right) \frac{d K(u)}{u^{2}}
$$


where $c$ is a constant and $K(u)$ is a non-decreasing bounded function, $K(-\infty)=$ 0 . The finding of $K(u)$ is based on the inversion of the Fourier transform

$$
-\frac{d^{2}}{d t^{2}} \log g_{2}(t)=\int_{-\infty}^{\infty} e^{i t u} d K(u)
$$

However,

$$
-\frac{d^{2}}{d t^{2}} \log g_{2}(t)=-\frac{3}{(i+t)^{2}}+b^{2}\left(\frac{1}{(-1+i+b(i+t))^{2}}+\frac{1}{(1+i+b(i+t))^{2}}\right) .
$$

Inverting Fourier transform we find

$$
d K(x)=e^{-x}\left(3-2 e^{-x / b} \cos (x / b)\right) \text { for } \quad x>0
$$

and 0 otherwise. The statement follows now from Kolmogorov representation.

Although we considered the case of shifted exponential distribution it is likely the similar hold for Gamma-distribution in general case.

The next example is connected to characteristic function of the negative binomial distribution

$$
f(t, r, p)=\left(\frac{p}{1-(1-p) e^{i t}}\right)^{r}, \quad 0<p<1, \quad r>0 .
$$

However, not all values of the parameters give suitable condition. Let us give a special example.

Example 3.3. Consider the function (3.4) for the case $p=1 / 2$. It has form

$$
f(t, r)=\left(2-e^{i t}\right)^{-r}, \quad r>0 .
$$

Corresponding $g_{2}(t, r)$ is

$$
g_{2}(t, r)=\frac{e^{i t}}{(r+1)\left(2-e^{i t}\right)^{r+1}}+\frac{e^{2 i t}}{\left(2-e^{i t}\right)^{r+2}}
$$

For the use of A.N. Kolmogorov representation we need its second logarithmic derivative:

$$
-\frac{d^{2}}{d t^{2}} \log g_{2}(t, r)=\frac{2 e^{i t}(1+r)\left(8+4 r e^{i t}+r(1+r) e^{2 i t}\right)}{\left(-2+e^{i t}\right)^{2}\left(2+r e^{i t}\right)^{2}}=
$$




$$
=\sum_{k=1}^{\infty} \frac{k\left(2+r-(-r)^{k}\right)}{2^{k}} e^{i k t}
$$

If all the coefficients of the last series are non-negative then its sum corresponds to non-decreasing function $K$. In the opposite case $g_{2}$ is not infinite divisible. It is clear that all the coefficients are non-negative if and only if $0<r \leq 1$.

Probably, the fact remains true for more general case of arbitrary $p \in$ $(0,1)$ and $g_{2 k}$.

Let us give one more example.

Example 3.4. Define

$$
f(t)=\exp \left\{\frac{1}{1-i t}-1\right\}=\exp \left\{\frac{i t}{1-i t}\right\}
$$

Obviously, $f(t)$ is infinite divisible characteristic function. Corresponding $g_{2}(t)$ has the following form

$$
g_{2}(t)=\frac{e^{-t /(i+t)}(2 i t-3)}{(i+t)^{4}} .
$$

$g_{2}(t)$ is infinite divisible function.

Proof. Apply A.N. Kolmogorov representation again. As it was mentioned above, it is sufficient to define corresponding function $K(x)$ by its Fourier transform:

$$
\int_{-\infty}^{\infty} e^{i t x} K(x)=-\frac{d^{2}}{d t^{2}} \log g_{2}(t)
$$

We have

$$
-\frac{d^{2}}{d t^{2}} \log g_{2}(t)=-\frac{2 i}{(i+t)^{3}}-\frac{4}{(i+t)^{2}}+\frac{4}{(3 i+2 t)^{2}}
$$

Applying inverse Fourier transform we obtain

$$
\frac{d K}{d x}=x e^{-3 x / 2}\left((4+x) e^{x / 2}-1\right) \quad \text { for } \quad x \geq 0
$$

and 0 for $x<0$.

Given Examples shows that for $g_{2 k}$ to be infinite divisible $f(t)$ must have singularities. The types of them may be essentially different. The problem of full description corresponding functions remain open. 


\section{Acknowledgment}

The work was partially supported by Grant GAČR 19-04412S.

\section{References}

[1] Ju. V. Linnik and I. V. Ostrovskii (1977). Decomposition of Random Variables and Vectors. American Mathematical Society Providence, Rhode Island.

[2] W. K. Hayman (1968) Meromorphic Functions. Oxford University Press, Ely House, London. 